\title{
Evaluation of trauma resources in rural northern Alberta identifies opportunities for improvement
}

\author{
Henry Y. Jiang, MD, PhD \\ Alyssa MacLean, MD \\ Jenny Yoon, MD \\ Susan Hughes, RN \\ Michael J. Kim, MD \\ Ram V. Anantha, MD, MSc \\ Sandy L. Widder, MD, MSc; \\ on behalf of the Edmonton Zone \\ Trauma and Acute Care \\ Collaborative (EZ-TRACC)
}

Accepted Dec. 5, 2019

\author{
Correspondence to: \\ S. Widder \\ 2D4 WMC, 8440-112 St NW \\ Edmonton AB T6G 2B7 \\ sandy.widder2@ahs.ca
}

DOI: $10.1503 /$ cjs.007119

\begin{abstract}
Background: The care of rural trauma patients in northern Alberta can be extremely challenging because of the vast geographic area, the limited access to health care facilities and the lack of adequate resources to manage severe injuries. Identifying gaps in equipment and personnel in rural centres can provide opportunities for improving the care of injured patients in these environments. We conducted a survey based on Canadian Accreditation Council quality indicators to evaluate trauma infrastructure and human resources in rural centres across northern Alberta.
\end{abstract}

Methods: A standardized survey was developed to assess the availability of trauma-specific equipment and personnel across the prehospital and emergency department (ED) settings. The survey was distributed to 50 peripheral hospitals biannually from January 2017 to September 2018. Two-tailed paired $t$ tests were used to evaluate changes in survey responses; a $p$ value of less than 0.05 was considered statistically significant.

Results: The survey response rate was $100 \%$. By the end of the study period, there were significant improvements in the number of providers $(p=0.04)$, nurses $(p=0.01)$ and dedicated trauma resuscitation bays $(p=0.04)$ in the ED for managing injured patients. There were also significant increases in the availability of equipment, including advanced airway management tools $(p=0.02)$, rapid infusion devices $(p=0.02)$ and warmers $(p=0.04)$. Access to $x$-ray equipment $(p=0.03)$ and computed tomography (CT) scanners $(p=0.04)$ as well as equipment to support telehealth and teleconferencing $(p=0.04)$ increased during the study period. Access to, and supply of, blood products also increased significantly $(p=0.02)$ during the study period.

Conclusion: Our study demonstrates that the trauma resources of rural health care centres may be evaluated in a standardized fashion centres, and the results point to opportunities to remedy gaps in equipment and personnel. Our methods may be applied to any trauma network that serves geographically large areas with a sparse distribution of health care facilities, to provide critical information for the optimization of resources in rural trauma.

Contexte : Les soins aux patients victimes de traumatismes en région rurale dans le nord de l'Alberta peuvent être très difficiles en raison de la superficie de la région, de l'accès limité aux établissements de santé et du manque de ressources pour soigner adéquatement les blessures graves. En repérant les lacunes en équipement et en personnel dans les établissements en région rurale, on peut créer des occasions d'améliorer les soins aux patients blessés dans ces milieux. Nous avons mené un sondage fondé sur les indicateurs de qualité du Conseil d'accréditation canadien pour évaluer les infrastructures et les ressources humaines en traumatologie dans les établissements des régions rurales du nord de l'Alberta.

Méthodes : Un sondage standardisé a été créé pour évaluer la disponibilité des équipements et des ressources humaines en traumatologie en contexte préhospitalier et aux services d'urgence. Le sondage a été distribué 2 fois par année à 50 hôpitaux entre janvier 2017 et septembre 2018. Des tests $t$ appariés ayant une hypothèse non nulle ont été utilisés pour évaluer les changements dans les réponses au sondage; les résultats ayant une valeur $p<0,05$ étaient considérés comme statistiquement significatifs.

Résultats : Le taux de participation au sondage était de $100 \%$. À la fin de la période étudiée, il y avait une amélioration significative du nombre de fournisseurs $(p=0,04)$, de personnel infirmier $(p=0,01)$ et d'espaces de réanimation réservés à la traumatologie $(p=0,04)$ dans les services d'urgence. Il y avait aussi une augmentation significative 
de la disponibilité de l'équipement, notamment des outils de prise en charge avancée des voies respiratoires $(p=0,02)$, des appareils de perfusion rapide $(p=0,02)$ et d'armoires chauffantes $(p=0,04)$. Les équipements de radiographie $(p=0,03)$ et de tomographie par ordinateur $(p=0,04)$ ainsi que les équipements facilitant la télémédecine et les téléconférences $(p=0,04)$ sont devenus plus accessibles pendant la période étudiée. Les réserves de produits sanguins et l'accès à ces produits a aussi augmenté de manière significative $(\mathrm{p}=0,02)$.

Conclusion : Notre étude montre que les ressources en traumatologie dans les établissements de santé en région rurale peuvent être évaluées de manière standardisée, et les résultats indiquent qu'il y a des occasions de combler les lacunes en équipement et en personnel. Notres méthodes peuvent être reproduites dans tout réseau de traumatologie couvrant un grand territoire où les établissements de santé sont dispersés, pour fournir des données critiques sur l'organisation des ressources de traumatologie en région rurale.

$\mathbf{T}$ raumatic injuries are the fifth leading cause of mortality in the province of Alberta, with more than 900 deaths (close to $5 \%$ of all deaths) per year. ${ }^{1-3}$ Albertans living in rural communities (areas with fewer than 25000 inhabitants) ${ }^{3}$ make up $22 \%$ of the population. ${ }^{2,3}$ Of these, $17 \%$ reside in communities in northern Alberta, ${ }^{4}$ a vast geographic area that spans over half a million square kilometres., ${ }^{2,3}$ Patients from rural areas may sustain injuries from agricultural trauma (such as machinery runovers, and entanglement and impingement by heavy equipment), motor vehicle collisions and animal encounters. ${ }^{3,5,6}$ However, management of these cases poses particular challenges for several reasons: long transport times owing to the diverse terrain and seasonal weather changes, variation in the expertise of the people providing prehospital care, ${ }^{7-9}$ the dearth of rural health care facilities, ${ }^{6}$ limited access to diagnostic equipment and medical specialists, and rural health care providers' infrequent exposure to severe trauma and their consequent inexperience in treating such cases. ${ }^{10-12}$ These barriers lead to increased morbidity and mortality among rural trauma patients, who are twice as likely to die from their injuries as their urban counterparts. ${ }^{5,6}$

Because of the disparity in access to trauma care in rural northern Alberta, ${ }^{4}$ we sought to evaluate the ability of health care facilities to receive, triage, stabilize and transfer injured patients. Fifty hospitals serve the inhabitants of northern Alberta (Fig. 1), and these hospitals must comply with trauma standards and guidelines developed by the Canadian Accreditation Council. ${ }^{1,13}$ Trauma centres are designated levels 1 through 5 depending on the availability of particular equipment and personnel. Level 1 and 2 hospitals provide definitive care for severely injured patients (defined as those with an Injury Severity Score [ISS] higher than 12). Level 3 trauma centres can manage moderately and severely injured trauma patients in areas where there is limited rapid access to higher level trauma centres. However, they are expected to transfer severely injured patients to a level 1 or 2 site after the patient's condition is stabliized, owing to lack of access to subspecialty surgical services and resources.
Level 4 and 5 trauma centres have limited resources to provide emergent resuscitation for patients; they focus on stabilizing the condition of trauma patients before rapidly facilitating transfer to higher levels of care. ${ }^{14}$

We developed a survey based on the Canadian Accreditation Council quality indicators for regional trauma care, ${ }^{14}$ to identify opportunities to improve patient care within our northern Alberta trauma system. We also sought to determine whether regular use of the survey led to improvements in personnel, equipment and infrastructure over an 18-month study period.

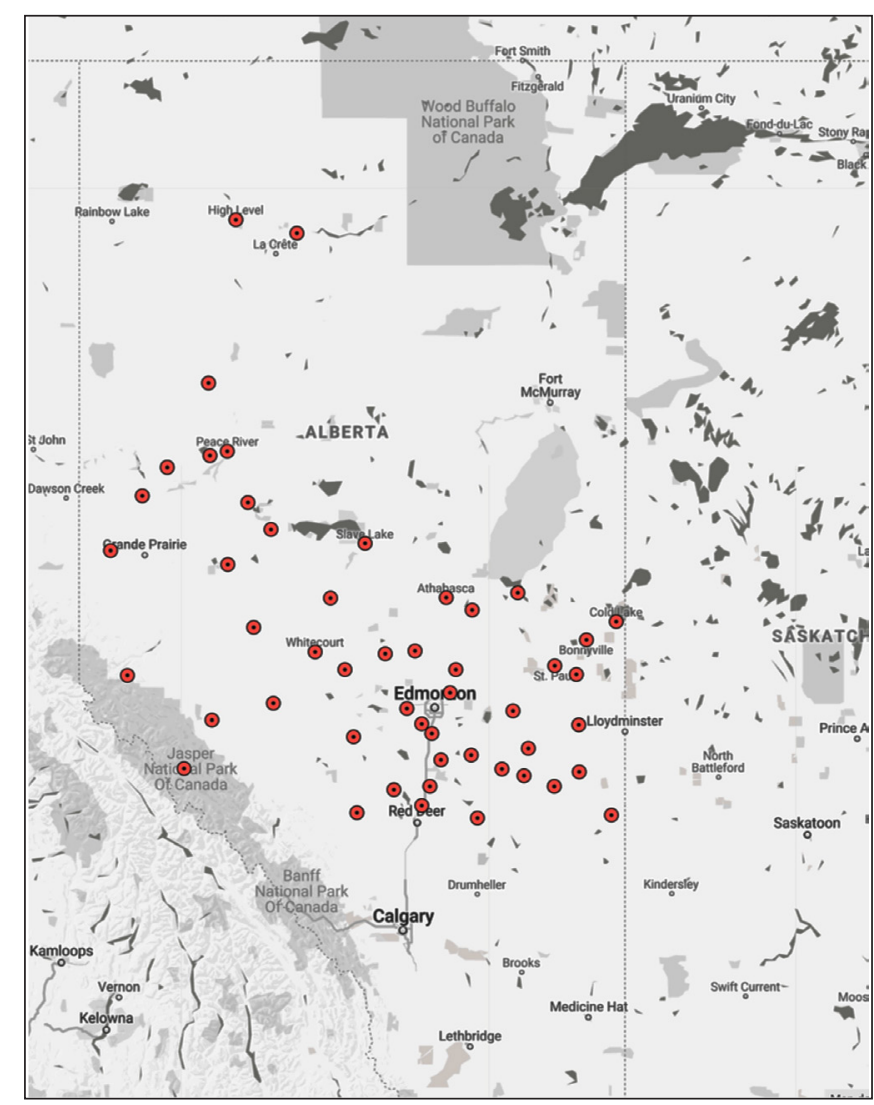

Fig. 1. Map showing the 50 health care facilities in northern Alberta that participate in the care of injured patients. 


\section{Methods}

\section{Study design}

This study was a prospective evaluation of hospital equipment and personnel, which was based on a survey distributed to the hospitals across Northern Alberta. The 2007 guidelines of Accreditation Canada were reviewed, and all criteria for accreditation including the qualifications of prehospital and trauma personnel, and trauma equipment, were extracted and formulated into questions for our survey (Appendix 1, Supplementary Table S1, available at canjsurg.ca/007119-a1).

The survey had 49 questions in 9 sections designed to identify both personnel and equipment needs at each of the hospitals. Key components of trauma care, including prehospital reception, the composition of the trauma team, individual team member accreditation/certification, inhospital equipment, radiology complement and posttrauma support services, were included.

Key stakeholders provided critical discussion and input for this study. They included medical and operational leaders in Alberta Health Services (AHS), Alberta Trauma Services (ATS), Referral, Access, Advice, Placement, Information \& Destination (RAAPID), Shock Trauma Air Rescue Service (STARS), Emergency Medical Services (EMS), physicians, nurses, care coordinators and managers.

Because this study did not involve any risk to participants, ethics approval from the University of Alberta Health Research Ethics Board was not required, as per the A pRoject Ethics Community Consensus Initiative (ARECCI) criteria.

\section{Participants}

Physician operational leads and nursing emergency managers from the 50 health care facilities in northern Alberta were invited to participate in the survey. The initial survey was distributed in January 2017, and updated versions were distributed every 6 months thereafter, until September 2018.

\section{Statistical analysis}

Results were compiled using Microsoft Excel. Descriptive statistics were calculated for all facilities. Categorical variables were reported as frequencies and percentages. Variables were compared using the paired $t$ test.

To characterize the geographic distribution of hospitals in northern Alberta, we geocoded the hospital addresses using BatchGeo, ${ }^{15}$ a geocoding service that implements the Google Maps geocoding application programming interface (API).

All tests were 2 -tailed, and a $p$ value $<0.05$ was considered to be statistically significant. Statistical analysis was performed using SPSS version 23 (IBM).

\section{Results}

Our survey response rate increased from 94\% (47/50) to $100 \%$ over the course of the study. Initially, 3 of the 50 hospitals did not participate because of a lack of coordinators at their sites. However, they were able to participate in the last 2 surveys, and the response rate for these surveys was therefore $100 \%$. Of the 50 participating facilities, 4 were classified as level 4 trauma centres and 46 as level 5 trauma centres (Appendix 1, Supplementary Table S2) according to the 2007 criteria of Accreditation Canada. ${ }^{14}$

There were a median of 2 ambulances (air and land) at each hospital, and this value did not change over the study period (Table 1). Ambulance crew certification in basic life support (BLS), however, increased significantly from $60 \%$ to $70 \%(p=0.04)$ across the study period (Table 1$)$. Seventy-eight percent of hospitals were able to deploy a STARS air ambulance in 2017 compared with $82 \%$ in $2018(p=0.16)$ (Table 1). Sixty percent of hospitals were equipped with a dedicated helipad in 2017, and this increased to $66 \%$ in 2018 , although the difference was not statistically significant (Table 1).

The availability of emergency department (ED) personnel improved significantly over the study period (Table 2). Provider availability within 30 minutes increased from $88 \%$ to $96 \%$ ( $p=0.04)$, while ED nursing coverage increased from $58 \%$ to $76 \%(p=0.01)$. We also observed a significant increase in the availability of appropriately trained personnel for the performance of emergency airway interventions ( $84 \%$ to $92 \%, p=0.04$ ) (Table 2), and a concomitant increase in the availability of advanced airway management equipment from $82 \%$ to $92 \%(p=0.02)$ as well (Table 3). Tourniquets, on the other hand, were available in only $74 \%$ of the hospitals at the end of the study period (Table 3 ).

The trauma bay in every hospital also had access to central, peripheral and intraosseous vascular access, chest tubes and Broselow tapes for pediatric trauma (Table 3). Although the availability of fracture stabilization equipment increased from $88 \%$ to $94 \%$ at the conclusion of our

Table 1. Comparison of prehospital transfer capabilities across the trauma system in northern Alberta between January 2017 and September 2018

\begin{tabular}{lccc|}
\hline Variable & $\begin{array}{c}\text { January } \\
2017\end{array}$ & $\begin{array}{c}\text { September } \\
2018\end{array}$ & $p$ value \\
\hline $\begin{array}{l}\text { No. of available EMS } \\
\text { ambulances, median (range) }\end{array}$ & $2(0-3)$ & $2(0-3)$ & \\
\hline EMS crew BLS certification, \% & 60 & 70 & 0.04 \\
\hline EMS crew ACLS certification, \% & 62 & 72 & 0.06 \\
\hline Ability to auto launch STARS, \% & 78 & 82 & 0.16 \\
\hline Helipad for STARS access, \% & 60 & 66 & 0.18 \\
\hline $\begin{array}{l}\text { ACLS = Advanced Cardiovascular Life Support; BCLS = Basic Life Support; EMS = } \\
\text { Emergency Medical Services; STARS = Shock Trauma Air Rescue Service. }\end{array}$
\end{tabular}




\begin{tabular}{|c|c|c|c|}
\hline Variable & $\begin{array}{l}\text { January } \\
2017\end{array}$ & $\begin{array}{l}\text { September } \\
2018\end{array}$ & $p$ value \\
\hline \multicolumn{4}{|l|}{ Emergency department } \\
\hline 24-h ED physician coverage, \% & 92 & 98 & 0.08 \\
\hline ED physicians with ATLS certification, \% & 34 & 36 & 0.32 \\
\hline $\begin{array}{l}\text { Proportion of providers with emergency airway } \\
\text { management training/certification, \% }\end{array}$ & 84 & 92 & 0.04 \\
\hline $\begin{array}{l}\text { 24-h availability to provide emergency airway } \\
\text { intervention, \% }\end{array}$ & 84 & 90 & 0.18 \\
\hline Provider availability within $30 \mathrm{~min}, \%$ & 88 & 96 & 0.04 \\
\hline Facilities with adequate ED nursing coverage, $\%$ & 58 & 76 & 0.01 \\
\hline ED nurses with TNCC certification or equivalent, \% & 50 & 54 & 0.32 \\
\hline No. of ED beds, median & 7 & 7 & NA \\
\hline \multicolumn{4}{|l|}{ Radiology access } \\
\hline Board-certified radiologist on staff, \% & 26 & 28 & 0.66 \\
\hline Available within $30 \mathrm{~min}, \%$ & 22 & 32 & 0.06 \\
\hline Conventional radiology available $24 \mathrm{~h}, \%$ & 78 & 90 & 0.03 \\
\hline CT available 24 h, \% & 12 & 20 & 0.04 \\
\hline Access to tele-radiology, $\%$ & 56 & 60 & 0.32 \\
\hline \multicolumn{4}{|l|}{ Anesthesia and medicine } \\
\hline $\begin{array}{l}\text { Access to board-certified anesthesiologist or certified } \\
\text { RN anesthetist, \% }\end{array}$ & 36 & 38 & 0.32 \\
\hline Access to internal medicine physician, $\%$ & 18 & 28 & 0.06 \\
\hline Access to family physician, $\%$ & 80 & 88 & 0.04 \\
\hline \multicolumn{4}{|l|}{ Laboratory access } \\
\hline 24-h access to laboratory services, \% & 86 & 90 & 0.32 \\
\hline 24-h access to blood bank, \% & 62 & 66 & 0.42 \\
\hline Massive transfusion protocol established, \% & 24 & 22 & 0.66 \\
\hline Adequate supply of blood products, $\%$ & 66 & 56 & 0.06 \\
\hline \multicolumn{4}{|l|}{ Allied health professions } \\
\hline Physical therapist, \% & 76 & 84 & 0.10 \\
\hline Social worker, \% & 60 & 62 & 0.57 \\
\hline Occupational therapist, \% & 76 & 80 & 0.42 \\
\hline Speech/language pathologist, \% & 58 & 68 & 0.06 \\
\hline \multicolumn{4}{|l|}{ Teleconferencing } \\
\hline Telehealth, \% & 92 & 100 & 0.04 \\
\hline Teleconferencing, \% & 90 & 98 & 0.04 \\
\hline Web conferencing, \% & 80 & 84 & 0.32 \\
\hline
\end{tabular}

study, traction equipment was only available in $36 \%$ of hospitals (Table 3). Some centres relied on loans of traction equipment from EMS crews. Other equipment that was considered desirable in the Accreditation Canada guidelines was universal precautions wear $(92 \%$ to $100 \%$, $p=0.04$ ), provision of a dedicated room in the ED for trauma resuscitation $(86 \%$ to $94 \%, p=0.04$ ), rapid infusion warmers $(56 \%$ to $66 \%, p=0.02)$, equipment for $\mathrm{ED}$ surgical procedures $(62 \%$ to $66 \%, p=0.32)$, vascular Doppler (52\% to $62 \%, p=0.02)$ and equipment for ultrasonography (unchanged at 70\%) (Table 3, Table 4).

The proportion of physicians and nurses with traumaspecific training and skill sets did not improve over the study period: $34 \%$ of hospitals had physicians trained in advanced trauma life support in 2017, compared with $36 \%$ in 2018; and $50 \%$ of hospitals had nurses with Trauma Nursing Core Course certification or an equivalent in 2017, compared with 54\% in 2018 (Table 2). Additionally, fewer than one-third of the hospitals had around-theclock access to specialty-certified radiologists and less than $40 \%$ of hospitals had access to anesthesiologists or nurse anesthetists at the start of our study (Table 2). This did not change during the study period. Less than $20 \%$ of hospitals had access to an internist in 2017; there was a small but not significant increase to $28 \%$ in 2018 ( $p=$ 0.06 ) (Table 2). Access to family physicians and hospitalists did improve significantly from $80 \%$ to $88 \%(p=0.04)$ (Table 2). Lastly, there was some improvement in the availability of other supportive services such as physiotherapy, occupational therapy, speech language pathology 
Table 3. Comparison of availability of and access to trauma equipment in emergency departments in northern Alberta between January 2017 and September 2018

\begin{tabular}{|lccc|}
\hline Variable & January 2017 & September 2018 & $p$ value \\
\hline $\begin{array}{l}\text { Advanced airway management equipment (adult and } \\
\text { pediatric), \% }\end{array}$ & 82 & 92 & 0.02 \\
\hline Broselow tape, \% & 94 & 100 & 0.08 \\
\hline $\begin{array}{l}\text { Central and peripheral vascular access equipment } \\
\text { (including IO), \% }\end{array}$ & 92 & 98 & 0.08 \\
\hline Chest tubes (all sizes), \% & & 98 & 0.08 \\
\hline Fracture stabilization, \% & 92 & 94 & 0.08 \\
\hline Traction equipment, \% & 88 & 36 & 0.08 \\
\hline Portable/overhead x-ray, \% & 36 & 88 & 0.08 \\
\hline Monitors (transport, bedside, fetal), \% & 82 & 82 & 0.04 \\
\hline Adequate supply of crystalloids and colloids, \% & 76 & 98 & 0.02 \\
\hline Rapid infusion warmer, \% & 90 & 66 & 0.32 \\
\hline Surgical equipment (DPL, thoracotomy tray), \% & 56 & 66 & 0.57 \\
\hline Tourniquets, \% & 62 & 74 & 0.04 \\
\hline Trauma resuscitation room, \% & 72 & 94 & 100 \\
\hline Universal precautions equipment, \% & 86 & 62 & 0.04 \\
\hline Vascular Doppler, \% & 92 & 96 & 0.02 \\
\hline Warming devices (Bair Hugger, blanket warmer), \% & 82 & 70 & 0.04 \\
\hline Ultrasonography, \% & 70 & & 1.00 \\
\hline DPL = diagnostic peritoneal lavage; IO = intraosseous. & & & \\
\hline
\end{tabular}

Table 4. Evaluation of trauma-specific personnel and equipment in level 4 and level 5 hospitals in northern Alberta between January 2017 and September 2018

\begin{tabular}{|c|c|c|c|c|}
\hline \multirow[b]{2}{*}{ Variable } & \multirow[b]{2}{*}{ January 2017} & \multirow[b]{2}{*}{ September 2018} & \multicolumn{2}{|c|}{$p$ value } \\
\hline & & & $\begin{array}{l}\text { Level } 4 \text { and } 5 \\
\text { hospitals }\end{array}$ & $\begin{array}{l}\text { Level } 5 \\
\text { hospitals only }\end{array}$ \\
\hline Adequate nursing coverage, $\%$ & 58 & 76 & 0.01 & 0.02 \\
\hline No. of beds in ED, median & 6.98 & 7.36 & 0.02 & 0.02 \\
\hline $\begin{array}{l}\text { Advanced airway management equipment (adult and } \\
\text { pediatric), \% }\end{array}$ & 82 & 92 & 0.02 & 0.04 \\
\hline Adequate supply of crystalloids and colloids, \% & 90 & 98 & 0.04 & 0.04 \\
\hline Rapid infusion warmer, \% & 56 & 66 & 0.02 & 0.02 \\
\hline Trauma resuscitation room, \% & 86 & 94 & 0.04 & 0.04 \\
\hline Universal precautions equipment, \% & 92 & 100 & 0.04 & 0.04 \\
\hline Vascular Doppler, \% & 52 & 62 & 0.02 & 0.02 \\
\hline Warming devices (Bair Hugger, blanket warmer), \% & 88 & 96 & 0.04 & 0.04 \\
\hline Crew BCLS certification, \% & 60 & 70 & 0.04 & 0.04 \\
\hline CT available 24 h, \% & 12 & 20 & 0.04 & 0.04 \\
\hline Access to internal medicine physician, \% & 18 & 28 & 0.06 & 0.04 \\
\hline Access to family physician, \% & 80 & 88 & 0.04 & 0.04 \\
\hline Adequate supply of blood products, \% & 66 & 56 & 0.06 & 0.02 \\
\hline Telehealth, \% & 92 & 100 & 0.04 & 0.04 \\
\hline Teleconferencing, \% & 90 & 98 & 0.04 & 0.04 \\
\hline
\end{tabular}

and social work during the study period (Table 2), but this was not statistically significant.

Twenty-four percent of the surveyed sites had defined massive transfusion protocols in 2017, with no statistical change in 2018 (Table 2). Sixty-six percent of the sites had an adequate supply of blood products onsite, although this proportion dropped to $56 \%$ at the end of the study period (Table 2). Twenty-four hour access to the hospital blood bank, however, remained above $60 \%$.

All centres had telehealth (100\%), teleconferencing $(98 \%)$ and web conferencing $(84 \%)$ capabilities at the end of the study period (Table 2). Telehealth was not available in the trauma bay for live guidance of emergent trauma resuscitations by trauma specialists from larger centres. 


\section{Discussion}

In this study, we developed a survey that incorporated critical questions to address trauma resource availability in northern Alberta. There was complete participation across our regional trauma system. Our survey was able to identify site-specific opportunities to enhance equipment and personnel, leading to improvements in delivery of trauma patient care. Our method of assessment may be applicable to similar trauma networks that are distributed over large geographic areas with varied levels of trauma centres.

We believe our high response rate resulted from the engagement of the communities by our trauma program manager, who reached out to each site personally and had one-on-one conversations with individuals at each site to discuss the premise of the study and its importance. Additionally, we were heading toward evaluation as a province and pursuing Accreditation with Distinction with Accrediation Canada; therefore, there was leadership buy-in and support for this questionnaire-based survey.

Although we did not actively allocate resources to help the hospitals surveyed, we surmise that the repeated distribution of surveys to stakeholders and trauma/emergency department coordinators stipulated quality improvement initiatives by providing data to these sites and by educating others (i.e., hospital leaders) about trauma standards of care, such as the use of tourniquets. Our survey may have acted as a change vehicle, expediting the process for improvement. Active feedback was not part of our study, as this was a quality initiative to determine current state and potential areas of improvement; this was a limitation of our study. Furthermore, our data were such that all results were surrogate outcomes or markers of preparedness. Hence, they did not in any way reflect on the actual quality of trauma care, which also depended on provider experience and training. Other systems can replicate these findings by ensuring appropriate stakeholder analysis and engagement before any quality improvement initiative, as well as rigorous data collection and transparency of data display.

One of the central areas of focus of our survey was prehospital resuscitation and transportation, given the strong association between short prehospital transportation time and survival among injured patients. ${ }^{16-19}$ Our current trauma network enables more than $80 \%$ of Albertans to access a trauma centre by land within 1 hour being injured. ${ }^{2,12,20,21}$ However, approximately $10 \%$ of rural communities in northern Alberta lie outside this guideline, ${ }^{12}$ highlighting the challenges in covering the vast geography adequately. Even if closer, but potentially less wellequipped, hospitals are bypassed en route to facilities that provide higher levels of care, $, 17,22$ it is not realistic to expect to transport patients directly to a level 1 trauma centre within 1 hour of injury in our system. Weather, geography and aircraft landing and refuelling specifications profoundly influence transport time. Even though $85 \%$ of the hospitals in this study have the ability to launch STARS, some sites, such as Cold Lake, may be so remote that refuelling and long-range air transportation may not be feasible at certain times of the year. Additionally, 26\% of hospitals do not have a helipad or open airfield for access to STARS. Consequently, ATS specifies time estimates rather than distance measures to evaluate transfer times from rural hospitals to level 1 or 2 trauma centres: 3 hours for distances less than $200 \mathrm{~km}, 4$ hours for distances between 200 and $400 \mathrm{~km}$ and 6 hours for distances greater than $400 \mathrm{~km} .{ }^{23}$ Despite these barriers, improvements in EMS training and increases in the number of medical transportation vehicles are encouraging signs for trauma coverage across the region. ${ }^{24}$ Although our survey did not include an assessment of the availability of fixedwing aircraft or the use of helicopters and/or fixed-wing aircraft to transport trauma patients, future studies evaluating the efficiency and coordination of land and air transport modalities across northern Alberta may provide substantial insight into prehospital transportation algorithms for severely injured patients. ${ }^{24}$

The results of our survey also demonstrate deficiencies in trauma-specific personnel training and equipment availability. Trauma training was lacking among ED providers in more than half of all hospitals surveyed. Sixty-two percent of ED physicians had ATLS certification, and $77 \%$ of ED nurses had TNCC training. In previous studies, rural physicians have expressed interest in managing patients with pediatric, orthopedic, vascular and blunt thoracic or abdominal trauma. ${ }^{25}$ Although courses such as ATLS and the Rural Trauma Team Development Course (RTTDC) may bridge these knowledge gaps, it may not be realistic or feasible to take health care providers away from the sparsely served rural sites they service to provide training. Our study did not examine differences in hospitals that have RTTDC personnel at any point during or before the study period. This will be of interest in future studies, and it also speaks to the importance of education and outreach in helping to promote trauma care at nontrauma sites. Given that the advantages of RTTDC are that training may be brought to the rural site and that it involves both physician and nonphysician health care professionals, our provincial body has begun teaching this course in multiple rural hospitals.

Real-time support through telemedicine has been proposed as a solution to this dilemma. Telemedicine empowers rural physicians to resuscitate injured patients with real-time aid from a trauma surgeon, thereby improving survival while patients are awaiting transfer. ${ }^{26,27}$ Moreover, provincewide electronic medical record infrastructure (Netcare and Impax) can provide trauma centre physicians with convenient access to laboratory and radiologic data for patients. Similarly, video and phone conferencing allow rural physicians to have ready access to trauma surgeons 
for patient management and educational sessions. ${ }^{28}$ Future studies examining the impact and utility of distance-based care will be critical to evaluate outcomes for rural trauma patients.

We also observed a lack of basic equipment including tourniquets and traction devices in EDs, which did not change substantially during the study period. Tourniquets are relatively inexpensive equipment that augment survival from extremity hemorrhage in civilian trauma. ${ }^{29,30}$ Consequently, they ought to be available and accessible to all rural EDs; these findings suggest that conducting courses on hemorrhage control, such as the American College of Surgeons' Stop the Bleed campaign, may be invaluable in promoting the use of tourniquets among health care providers.

Access to transfusion devices and blood products is also a critical priority for the resuscitation of trauma patients, although we recognize that it can be impractical for rural hospitals to carry large quantities of blood products that they may use only infrequently. The minimum amount of blood products that rural hospitals must have varies widely in northern Alberta. The distribution of blood and blood products is driven by the provincial Transfusion Medicine Committee and is based on resource utilization, cost and wastage. Although prompt transfusion of noncrossmatched blood is critical in resuscitating many injured patients, the focus within our regional trauma system is to prioritize timely transfer of patients to a higher level of care where more intensive blood product resuscitation and massive transfusion protocols can be implemented. Any trauma patient requiring blood or blood products should be transferred expeditiously to a trauma centre. We did not focus on the availability of hemostatic products with longer shelf lives, such as tranexamic acid and fibrinogen, even though they may provide temporary assistance with hemostasis while a patient is being prepared for transport; this presents an opportunity for future refinement of our survey.

\section{Conclusion}

Our survey increased awareness of resource deficiencies within our regional trauma network in rural northern Alberta. The results of our study demonstrate an inexpensive and feasible method of evaluating resource capacity within a geographically diverse trauma network with large numbers of rural inhabitants. Further refinement of the survey, as well as ongoing process and quality improvement assessments, will help to optimize the management of injured patients across the region.

Affiliations: From the Department of Surgery, University of Alberta Hospital, University of Alberta, Alta. (Jiang, MacLean, Yoon, Hughes, Kim, Anantha, Widder); and Alberta Trauma Services Edmonton Zone, Alberta Health Services, Edmonton, Alta. (Hughes, Kim, Anantha, Widder).
Competing interests: None declared.

Contributors: H. Jiang, A. MacLean, J. Yoon, S. Hughes and S. Widder designed the study. H. Jiang, A. MacLean, J. Yoon and S. Hughes acquired the data, which H. Jiang, A. MacLean, J. Yoon, S. Hughes, M. Kim and R. Anantha analyzed. H. Jiang, A. MacLean, J. Yoon and R. Anantha wrote the manuscript, which all authors critically revised. All authors gave final approval of the version to be published.

Members of the Edmonton Zone Trauma and Acute Care Collaborative (EZ-TRACC): Henry Jiang, Alyssa MacLean, Jenny Yoon, Susan Hughes, Michael J. Kim, Ram V. Anantha, Sandy L. Widder.

\section{References}

1. Calgary Health Regional. Regional Trauma Services: annual report. Calgary: Calgary Health Region; 2007. Available: https://www. albertahealthservices.ca/assets/info/hp/trauma/if-hp-trauma-calzone -ann-rpt-gen-07.pdf (accessed 2019 Feb. 12).

2. Alberta Government. Quarterly population report: first quarter 2018. Edmonton: Alberta Treasury and Finance; 2018.

3. Alberta Health Services. AHS map and zone overview. Available: https://www.albertahealthservices.ca/assets/about/publications/ahs-ar -2017/zones.html (accessed 2018 Nov. 12).

4. McCrum ML, McKee J, Lai M, et al. ATLS adherence in the transfer of rural trauma patients to a level I facility. Injury 2013;44:1241-5.

5. Alberta Centre for Injury Control and Research. Agricultural-related injuries in Alberta. Edmonton: Canadian Centre for Injury Control and Research; 2010. Available: https://injurypreventioncentre. ca/downloads/data/Alberta_CAISP_Deaths1990-2009_FINAL.pdf (accessed 2019 Feb. 12).

6. Bell N, Simons RK, Lakha N, et al. Are we failing our rural communities? Motor vehicle injury in British Columbia, Canada, 20012007. Injury 2012;43:1888-91.

7. Peek-Asa C, Zwerling C, Stallones L. Acute traumatic injuries in rural populations. Am f Public Health 2004;94:1689-93.

8. Kortbeek JB, Buckley R. Trauma-care systems in Canada. Injury 2003;34:658-63.

9. Malekpour M, Neuhaus N, Martin D, et al. Changes in rural trauma prehospital times following the Rural Trauma Team Development Course training. Am 7 Surg 2017;213:399-404.

10. Basak F. Addition of a general surgeon without addition of appropriate support is inadequate to improve outcomes of trauma patients in a rural setting: a cohort study of 1962 consecutive patients. Eur $\mathcal{F}$ Trauma Emerg Surg 2017;43:835-9.

11. Fedor PJ, Burns B, Lauria M, et al. Major trauma outside a trauma center: prehospital, emergency department, and retrieval considerations. Emerg Med Clin North Am 2018;36:203-18.

12. Hameed SM, Schuurman N, Razek T, et al. Access to trauma systems in Canada. 7 Trauma 2010;69:1350-61, discussion 1361.

13. Wiggins B, Hoogers K, Thomas L. Trauma Program annual report. Red Deer Regional Hospital: Central Zone. Red Deer: Central Zone Trauma Program; 2011. Available: https://www.albertahealthservices. ca/assets/info/hp/trauma/if-hp-trauma-cenzone-reddeer-ann-rpt-11.pdf (accessed 2019 Feb. 12).

14. Trauma Association of Canada. Trauma system accreditation guidelines. Calgary: Available: Trauma Association of Canada; 2011. Available: https://www.traumacanada.org/wp-content/uploads/2017/10/ Accreditation_Guidelines_2011-2.pdf (accessed 20 Mar. 2019).

15. Duncan DT, Castro MC, Blossom JC, et al. Evaluation of the positional difference between two common geocoding methods. Geospat Health 2011;5:265-73.

16. Liberman M, Mulder D, Sampalis J. Advanced or basic life support for trauma: meta-analysis and critical review of the literature. $f$ Trauma 2000;49:584-99.

17. Fahey M. Pre-hospital trauma management. NZ Med f 1978;87:54-8. 
18. Wilmink AB, Samra GS, Watson LM, et al. Vehicle entrapment rescue and pre-hospital trauma care. Injury 1996;27:21-5.

19. Alberta Health Services. EMS medical control protocols. Edmonton: Alberta Health Services, Emergency Medical Services, 2019. Available: http://protocols.ahsems.com/ (accessed 2019 Feb. 12).

20. Simons RK. Rural accidental injury and death: The neglected disease of modern trauma systems? 7 Trauma Acute Care Surg 2018;84:972-7.

21. Northern Alberta Development Council. Economic and demographic profile of Northern Alberta. Peace River (AB): Northern Alberta Development Council; 2005.

22. Fleet R, Tounkara FK, Ouimet M, et al. Portrait of trauma care in Quebec's rural emergency departments and identification of priority intervention needs to improve the quality of care: a study protocol. BM7 Open 2016;6:e010900.

23. VanWijngaarden-Stephens M. Royal Alexandra Hospital, University of Alberta Hospital and Stollery Children's Hospital 2013 trauma report. 2013. Edmonton (AB): Alberta Trauma Registry; 2013.

24. Uleberg O, Kristiansen T, Pape K, et al. Trauma care in a combined rural and urban region: an observational study. Acta Anaesthesiol Scand 2017;61:346-56.
25. Tarighi P, Sherman JE, Mian O, et al. The importance of tailoring physicians' trauma care training needs in rural environments. Can $\mathcal{F}$ Surg 2015;58:423-5.

26. Mohr NM, Vakkalanka JP, Harland KK, et al. Telemedicine use decreases rural emergency department length of stay for transferred North Dakota trauma patients. Telemed 7 E Health 2018;24:194-202.

27. Speyer R, Denman D, Wilkes-Gillan S, et al. Effects of telehealth by allied health professionals and nurses in rural and remote areas: a systematic review and meta-analysis. $\mathcal{F}$ Rebabil Med 2018;50:225-35.

28. Weiss BJ, Azevedo K, Webb K, et al. Telemental health delivery of Skills Training in Affective and Interpersonal Regulation (STAIR) for rural women veterans who have experienced military sexual trauma. 7 Trauma Stress 2018;31:620-25.

29. Cornelius B, Campbell R, McGauly P. Tourniquets in trauma care: a review of application. 7 Trauma Nurs 2017;24:203-7.

30. Beaucreux C, Vivien B, Miles E, et al. Application of tourniquet in civilian trauma: systematic review of the literature. Anaesth Crit Care Pain Med 2018;37:597-606. 